\title{
Divergence with gene flow across a speciation continuum of Heliconius butterflies
}

\author{
Megan A. Supple ${ }^{1,2,3}$, Riccardo Papa ${ }^{4}$, Heather M. Hines ${ }^{5}$, W. Owen McMillan ${ }^{1}$ and Brian A. Counterman ${ }^{6 *}$
}

\begin{abstract}
Background: A key to understanding the origins of species is determining the evolutionary processes that drive the patterns of genomic divergence during speciation. New genomic technologies enable the study of highresolution genomic patterns of divergence across natural speciation continua, where taxa pairs with different levels of reproductive isolation can be used as proxies for different stages of speciation. Empirical studies of these speciation continua can provide valuable insights into how genomes diverge during speciation.

Methods: We examine variation across a handful of genomic regions in parapatric and allopatric populations of Heliconius butterflies with varying levels of reproductive isolation. Genome sequences were mapped to 2.2-Mb of the $\mathrm{H}$. erato genome, including $1-\mathrm{Mb}$ across the red color pattern locus and multiple regions unlinked to color pattern variation.
\end{abstract}

Results: Phylogenetic analyses reveal a speciation continuum of pairs of hybridizing races and incipient species in the Heliconius erato clade. Comparisons of hybridizing pairs of divergently colored races and incipient species reveal that genomic divergence increases with ecological and reproductive isolation, not only across the locus responsible for adaptive variation in red wing coloration, but also at genomic regions unlinked to color pattern.

Discussion: We observe high levels of divergence between the incipient species H. erato and H. himera, suggesting that divergence may accumulate early in the speciation process. Comparisons of genomic divergence between the incipient species and allopatric races suggest that limited gene flow cannot account for the observed high levels of divergence between the incipient species.

Conclusions: Our results provide a reconstruction of the speciation continuum across the $H$. erato clade and provide insights into the processes that drive genomic divergence during speciation, establishing the $\mathrm{H}$. erato clade as a powerful framework for the study of speciation.

Keywords: Speciation genomics, Speciation continuum, Genomic divergence, Heliconius

\section{Background}

The ability to study high-resolution genomic patterns of divergence in natural populations has enabled valuable insights supporting a fundamental shift in our understanding of the origins of species. Historically, speciation research considered geographic isolation (allopatry) as the main barrier that allowed populations to diverge and reproductive incompatibilities to evolve. However, more recent theoretical and empirical studies have demonstrated that natural selection can be an important driver

\footnotetext{
* Correspondence: bcounterman@biology.msstate.edu

${ }^{6}$ Department of Biological Sciences, Mississippi State University, 295 Lee Boulevard, Mississippi State, MS 39762, USA

Full list of author information is available at the end of the article
}

of divergence and reproductive isolation, even when populations overlap geographically (parapatry or sympatry) (see [1] for a review). As a result, there has been a shift towards understanding how populations diverge as reproductive isolation increases, but gene flow continues to occur [2]. Genome scans between incompletely reproductively isolated populations and species offer snapshots of the genomic changes that accumulate during the speciation process. Valuable insights into how genomes diverge during speciation come from studies across speciation continua, where taxa pairs with different levels of reproductive isolation can be used as proxies for different stages of speciation. 
Initially, metaphorical models invoking selection and differential gene flow were proposed to explain the heterogeneous patterns of genomic divergence that occur as speciation progresses [3]. Two types of genetic hitchhiking, divergence hitchhiking and genomic hitchhiking, were introduced to explain how selection could affect levels of gene flow locally and globally across the genome [4]. Divergence hitchhiking is the result of selection acting on loci responsible for ecological divergence. This causes the local reduction of gene flow in physically linked regions, which leads to the further accumulation of allelic differences around the targets of selection. In genomic hitchhiking, the reduction of gene flow due to divergent selection reduces genome-wide rates of gene flow. This results in the global accumulation of genetic differences. While these two processes are similar and not mutually exclusive, they reflect two fundamentally different ways that genomes may evolve during speciation-through the local accumulation of divergence around targets of selection or through the genome-wide accumulation of divergence [4].

Heliconius butterflies offer an exceptional opportunity to explore patterns of divergence at different stages across the speciation continuum. The radiation of warning colors across the genus has resulted in an amazing diversity of wing color patterns within and between species. For example, Heliconius erato has radiated into over 25 geographically distinct color pattern races distributed throughout the Americas [5]. When these divergently colored races come into contact, they often hybridize. While races of $H$. melpomene have been shown to mate assortatively based on wing color patterns [6], this has not been demonstrated between races of $\mathrm{H}$. erato. Heliconius erato practices pupal mating, which involves males mating with partially eclosed females, thereby reducing the opportunity for wing color based mate choice [7]. Empirical support for the lack of color based assortative mating comes from hybrid zone analysis that shows Hardy-Weinberg equilibrium of wing color patterns [8]. Hybrid zones are characterized by the breakdown of parental color patterns, including the presence of individuals with intermediate color patterns created through multiple generations of backcrossing [8]. The high frequency of these hybrid phenotypes suggests that there is ongoing gene flow between $H$. erato races with overlapping geographic distributions. The taxonomic boundaries between hybridizing $H$. erato races are primarily maintained by strong frequency dependent selection on wing color patterns [9-13]. This selection is driven by Müllerian mimicry, as similarly unpalatable butterflies are more likely to be avoided if they exhibit a more common wing color pattern, which is more readily recognized by predators.
Besides natural selection due to differential predation based on wing color patterns, additional forms of reproductive isolation have been shown to maintain the species boundaries within Heliconius. For example, there is strong color pattern based mate preference between the closely related $H$. himera and $H$. erato cyrbia [14-18]. These species are associated with very different habitats that likely also contribute to ecological isolation: $H$. himera is found in very dry habitats at altitudes above $1000 \mathrm{~m}$; whereas, H. e. cyrbia is found in wetter forests at elevations below $1200 \mathrm{~m}$ [18]. Despite the strong premating isolation and habitat differences between these two species, hybrids comprise $5-10 \%$ of the population in the narrow zones of overlap [16-18]. Crosses between $H$. himera and several $H$. erato color pattern races have shown that F1 and backcrosses are reproductively viable, suggesting that intrinsic postzygotic barriers have not evolved between the species [14].

Previous studies of the evolutionary relationship between $H$. erato and H. himera have been equivocal. Early phylogenetic analysis using mtDNA placed the split between $H$. himera and $H$. erato prior to the $H$. erato radiation [19]. Additionally, a study of allozyme divergence between populations of $H$. himera and H. e. cyrbia found clear differences in allele frequencies, supporting the hypothesis that $H$. himera and $H$. erato are distinct species [20]. However, an analysis of rapidly evolving nuclear introns placed $H$. himera within the broader $H$. erato radiation, although with low support [21]. That study suggests that $H$. himera is a group of individuals that diverged during the $H$. erato radiation and over time became reproductively isolated from other $H$. erato populations.

There has been substantial progress resolving the genetic basis and evolution of the warning color radiation in Heliconius [22-28]. The color pattern variation across the genus is controlled by a handful of unlinked loci that control major differences in wing color patterns. In $H$. erato the adaptive radiation is largely controlled by allelic changes at three unlinked major color pattern loci $[29,30]$. The locus controlling red patterning (the $D$ locus) is the best characterized and controls the largest changes in warning color. Variation at this locus results in two major color pattern phenotypes: the postman phenotype, which has a red forewing band, and the rayed phenotype, which has a yellow forewing band, red proximal forewing patch, and red hindwing rays (Fig. 1). A combination of linkage analysis, gene expression, and genotype by phenotype association strongly implicate cis-regulatory changes in the transcription factor optix as causing variation in red wing pattern elements in Heliconius [23]. More recently, genome scans have narrowed the cis-regulatory region driving variation to a 65-kb region about 100-kb downstream of optix [31]. Phylogenetic analyses targeting this genomic region 


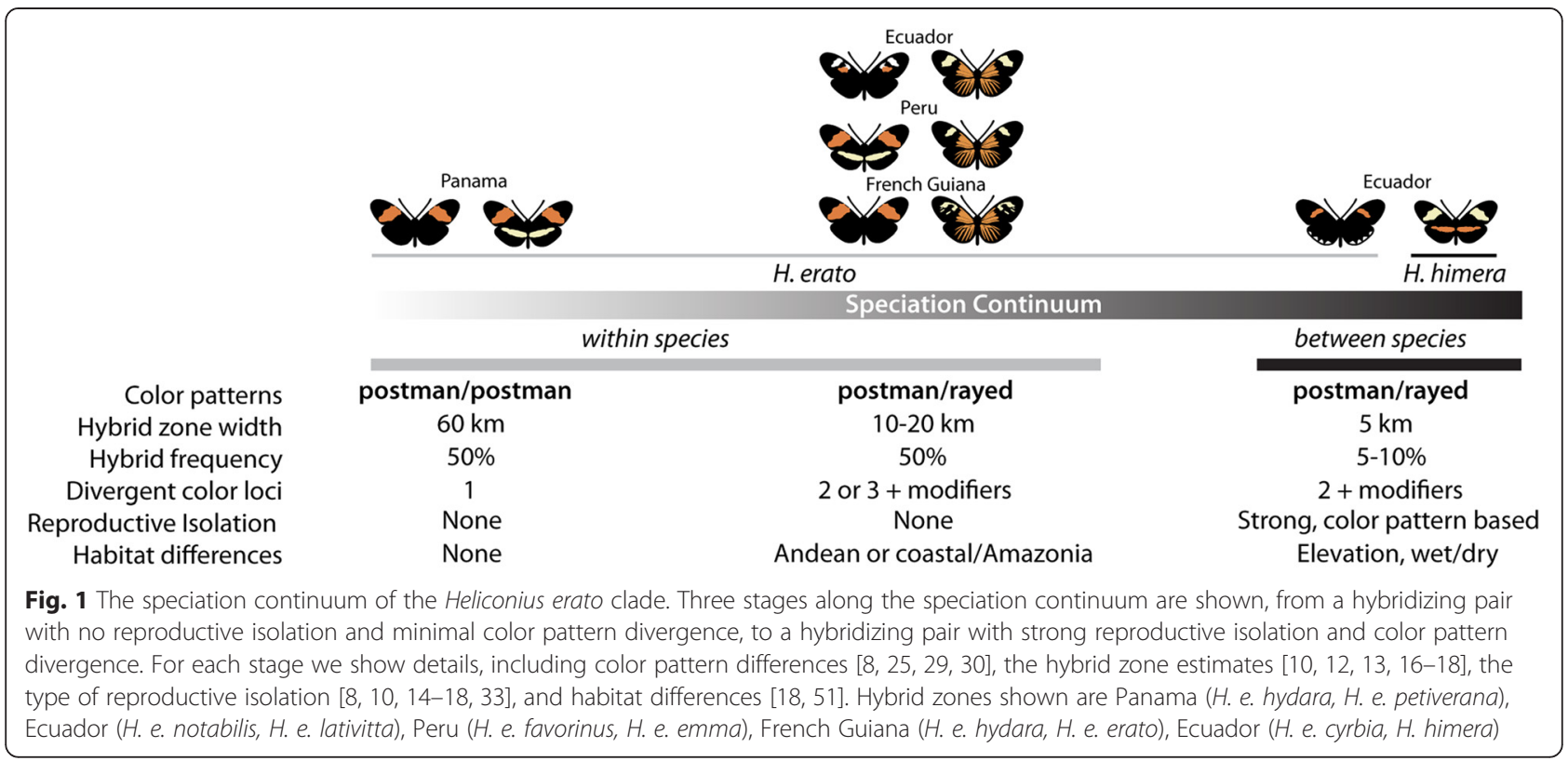

cluster $H$. erato races by color pattern, suggesting a single origin of the two major color pattern types within $H$. erato [31, 32].

Here, we leverage the recent progress in identifying the genomic region responsible for warning color variation to examine how genomes diverge through the speciation process. Specifically, we sample genomic variation across four geographically distinct hybrid zones between $H$. erato color pattern races and one hybrid zone between the proposed incipient species, $H$. himera and $H$. e. cyrbia. First, we reconstruct the evolutionary relationship of $H$. himera and races of $H$. erato. We use this phylogeny and a literature review of hybridization and reproductive barriers between $H$. erato races and $H$. himera to demonstrate the speciation continuum across the $H$. erato clade (Fig. 1). Then by sampling hybridizing races and species that reflect distinct points along this continuum, we empirically explore genomic divergence during the speciation process.

\section{Methods}

\section{Sampling the $\boldsymbol{H}$. erato speciation continuum}

We examined phenotypically pure samples from five hybrid zones across the $H$. erato radiation with varying degrees of reproductive isolation. The first four hybrid zones are all between hybridizing races of $H$. erato that show no evidence of pre-mating or post-mating isolation [8, 10, 33]. One of these hybrid zones is between two different postman phenotypes $(H$. e. hydara $\mathrm{x} H$. e. petiverana in Panama, $n=8)$. The other three are between divergent postman and rayed phenotypes $(H$. e. erato $\times H$. e. hydara in French Guiana, $n=13 ; H$. e. emma $\times H$. e. favorinus in Peru, $n=14$; and $H$. e. lativitta $\times H . e$. notabilis in Ecuador, $n=10$ ). For these four withinspecies hybrid zones, we utilized previously published data [GenBank:SRA059512] [31]. We have added to these data samples from a fifth hybrid zone between $H$. himera and $H$. erato cyrbia, two putative incipient species with pre-mating isolation, but no intrinsic post-mating isolation. We collected phenotypically pure samples of $H$. himera $(n=5)$ and $H$. e. cyrbia $(n=4)$ on either side of a narrow region of hybridization in Loja Province, Ecuador. We also collected samples from two outgroup species, $H$. clysonymus $(n=2)$ and $H$. telesiphe $(n=2)$ in Peru. Sampling locations are listed in Additional file 1: Table S1.

Within these intraspecific and interspecific hybrid zones, gene flow is known to occur based on the presence of hybrid individuals with wing color patterns that result from genetic backcrosses (e.g. individuals homozygous at one color pattern locus, but heterozygous at another) $[8,17]$. However, each hybrid zone sampled is geographically isolated from one another, with some on opposite sides of South America and separated by major topographic features. This geographic isolation severely restricts gene flow between the races from different hybrid zones.

\section{Sequencing and genotyping}

The samples from the $H$. himera/H. e. cyrbia hybrid zone and the outgroup taxa were sequenced and genotyped as described in [31]. Briefly, this involved whole genome sequencing of 100-bp paired end reads on the Illumina HiSeq platform and aligning reads to a partial genomic reference (2.2-Mb, $\sim 0.5 \%$ of the $H$. erato genome) [GenBank:KC469892-KC469895， AC208805-AC208806] with BWA v0.59-r16 [34] using relaxed mapping parameters. Hybrid zone samples were each sequenced to a 
realized median per base coverage between $13 x$ and $21 \times$ (Additional file 1: Table S1). Outgroup species samples were sequenced to a realized median coverage of $8 \mathrm{X}$ or higher, due to a lower proportion of sequence reads mapping to the $H$. erato reference. Multi-sample genotypes were then called and filtered with GATK v1.2 [35, 36]. Aligned sequencing reads are available at NCBI SRA [GenBank:SRA059521] and genotype VCF files are available at Dryad [doi:10.5061/dryad.5n10d]. We examined within species genetic variation using four previously published parapatric $H$. erato racial hybrid zones [GenBank:SRA059512] [31]. Sequence variation for the newly sequenced and previously sequenced samples ( $n=58$ combined) was analyzed across a $1-\mathrm{Mb}$ region containing the locus controlling red color pattern variation [GenBank:KC469894] and 350-kb from three additional genomic regions unlinked to color pattern loci [GenBank:KC469892, AC208805, AC208806].

\section{Phylogenetic analysis}

We performed phylogenetic analyses of all the genomic data to (i) determine the phylogenetic relationship of $H$. himera and $H$. erato, which has previously been disputed [19-21], and (ii) use the phylogenetic relationships to develop expectations for genetic divergence between taxa. We generated phylogenetic consensus trees from 5,399 SNPs across the previously identified 65-kb region that controls red color pattern variation [31] and 15,714 SNPs across the $350-\mathrm{kb}$ of genomic regions unlinked to color pattern. To reduce the size of the unlinked dataset, we removed invariant sites.

We selected nucleotide substitution models for both datasets using a hierarchical likelihood ratio test implemented in PAUP* v4 [37] and MrModeltest v2 [38]. We used MrBayes v3.2.2 [39] to generate consensus trees with the selected models-GTR + I+G for the $65-\mathrm{kb}$ functional region and GTR $+\mathrm{G}$ for the unlinked loci. For each tree, we ran 5 runs for 5 million generations each, sampling every 500 generations and removing $25 \%$ burn-in. We assessed burn-in and convergence in MrBayes and Tracer v1.6 [40]. For the unlinked tree, we removed two runs that converged to a slightly lower likelihood than the other three runs. We generated consensus trees from converged runs and rooted the trees with the $H$. clysonymus and $H$. telesiphe samples. Tree files are available at Dryad [doi:10.5061/dryad.5n10d]. We accounted for gene flow and phylogenetic conflict in inferring relationships by constructing an unrooted neighbor-net splits tree network on the unlinked dataset using SplitsTree v4.13.1 [41]. This analysis utilized pairwise distances and treated polymorphisms additively, rather than as ambiguities. We performed additional analyses to assess potential causes of the long branch lengths leading to the $H$. himera lineage. Specifically, we tested if the long branches leading to $H$. himera could be the result of different rates of evolution. We used BEAST v1.7.5 [42] to reconstruct the evolutionary relationships of $H$. himera and $H$. erato samples based on the 15,714 SNPs across the genomic regions unlinked to color pattern using i) a relaxed molecular clock and ii) a strict molecular clock. We used a GTR + I + G (four rate categories) model, estimated base frequencies, and a Yule tree prior. For each tree, we ran 2 runs for 10 million generations each, sampling every 5000 generations and removing $10 \%$ burn-in. We compared the likelihood distributions from the relaxed clock and the strict clock using BayesFactors (1,000 bootstrap replicates) in Tracer version 1.5 and visually compared the relaxed clock and the strict clock consensus phylogenies, each constructed from 18,000 sampled trees.

\section{Genomic divergence analysis}

We compared patterns of divergence between the incipient species, $H$. himera and $H$. e. cyrbia (between species comparison), to patterns of divergence among hybridizing races (within species, parapatric comparisons) and nonhybridizing races (within species, allopatric comparisons). The within species parapatric comparisons consisted of three $H$. erato hybrid zones between postman and rayed phenotypes (Peru, Ecuador, and French Guiana) and one $H$. erato hybrid zone between two different postman phenotypes (Panama). The within species allopatric comparisons consisted of all remaining pairwise comparisons between these same $H$. erato races, with the two Panamanian postman races being treated as a single race. These comparisons can be divided into three categories (postman versus rayed, $n=9$ comparisons; postman versus postman, $n=6$ comparisons; rayed versus rayed, $n=3$ comparisons). Note that $H$. e. cyrbia is only included in comparison to $H$. himera. Heliconius erato cyrbia is not included in the within $H$. erato comparisons due to interspecific hybridization and the possibility of divergent $H$. himera haplotypes in H. e. cyrbia individuals inflating the within species divergence estimates.

For each comparison, we calculated relative divergence per genomic position using a model for diploid data with populations as random effects $(\hat{\theta})$, a commonly used estimate of $F_{S T}$ [43], with implementation details described in [31]. In addition to relative divergence, for each comparison we also calculated absolute divergence $\left(d_{x y}\right)$ [44, equation 10.20]. For both analyses, we filtered data to remove positions where less than $75 \%$ of individuals were genotyped for each of the two taxa being compared. To properly combine estimates across loci and across comparisons for relative divergence, the numerators and denominators are each summed across the pairwise estimates and then divided to produce the final 
combined estimate. To combine estimates for absolute divergence, the mean was used. We calculated the genomic background level of relative divergence from three genomic intervals $(0.35-\mathrm{Mb})$ unlinked to color pattern, using 1000 bootstrap replicates to determine $95 \%$ confidence intervals (CIs). For sliding window analyses, we examined $15-\mathrm{kb}$ windows, with $5-\mathrm{kb}$ steps, requiring estimates for at least $20 \%$ of the positions in the window.

To examine the decay of divergence with recombination distance, we defined the causative locus as the center of the $65-\mathrm{kb}$ region previously identified to modulate red color pattern variation in $H$. erato [31]. We converted genomic distance (bp) to recombination distance (cM) assuming a constant recombination rate based on the $H$. erato linkage map size (1430 cM) [45] and the estimated size of the H. erato genome (400 Mb) [46], which provides an average estimate of recombination rate across the genome. Loci were binned by recombination distance from the causative locus in bins of size $0.01 \mathrm{cM}$. Decay data were loess smoothed for presentation.

\section{Results and Discussion}

Heliconius himera-an incipient species from within the $H$. erato radiation

Our phylogenetic analysis clearly places $H$. himera within the $H$. erato radiation, both at the color pattern locus and at loci unlinked to color pattern (Figs. 2 and 3 ). These results indicate that $H$. himera evolved from within the broader $H$. erato color pattern radiation, rather than predating it. The Bayesian phylogenetic tree of $H$. erato races across loci unlinked to color pattern shows a strong geographic signal, with no clustering by

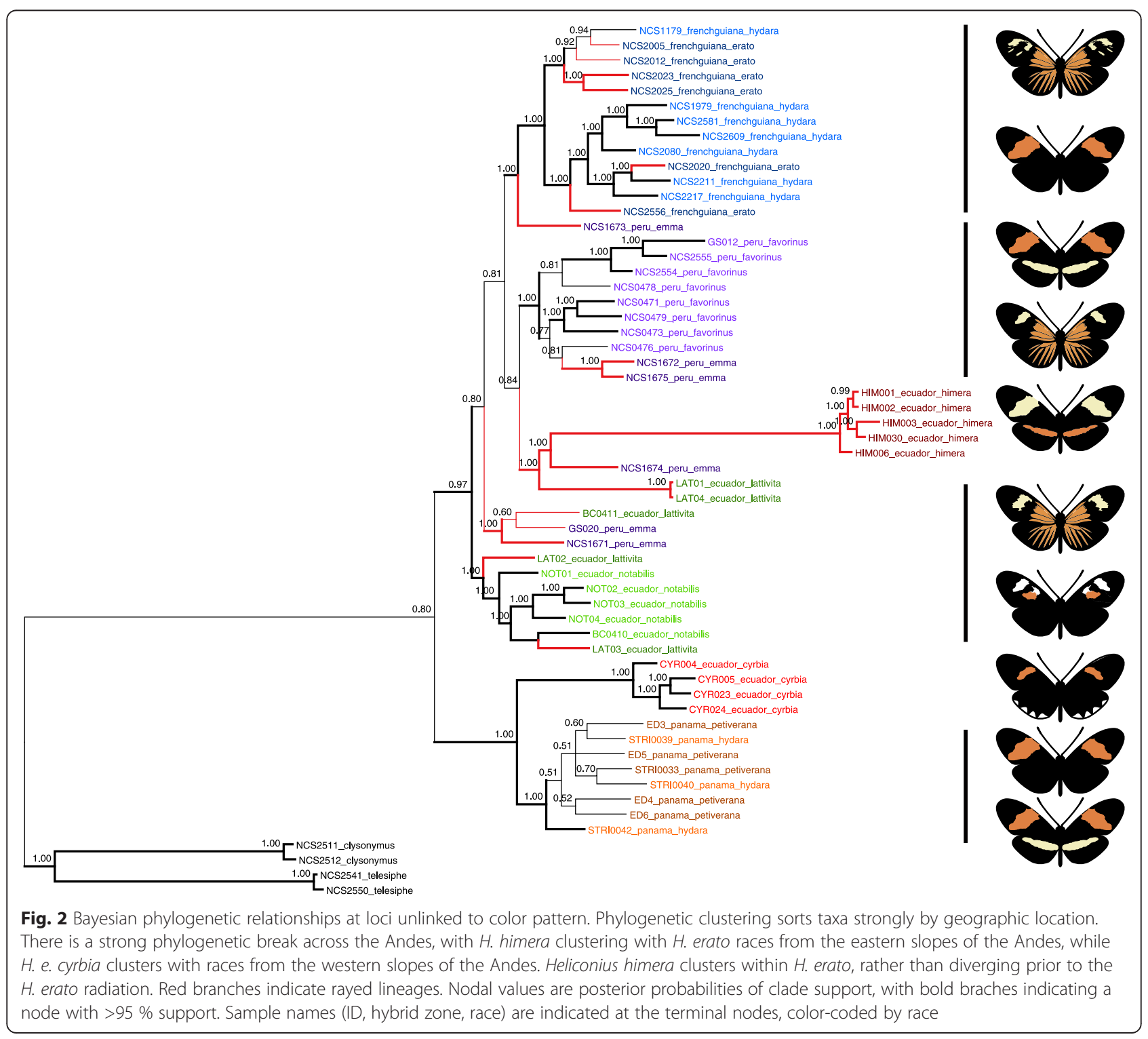




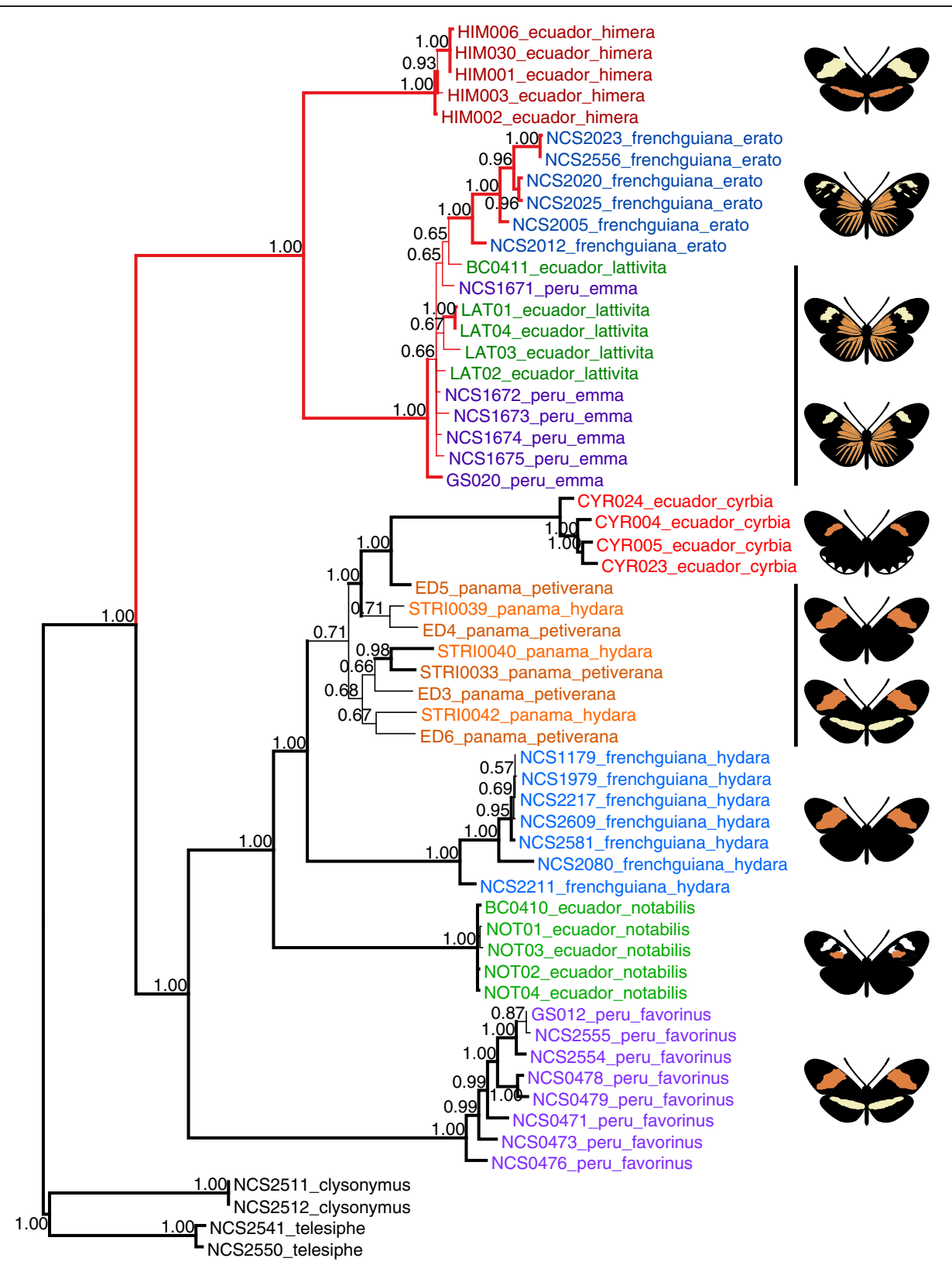

Fig. 3 Bayesian phylogenetic relationships at the red color locus. Phylogenetic clustering across the 65-kb region that modulates red color pattern variation sorts taxa by phenotype. The postman and the rayed clades form reciprocally monophyletic lineages, with $\mathrm{H}$. himera clustering with the rayed races, while H. e. cyrbia clusters with the postman races. Within each phenotype, samples cluster by geography. Heliconius himera clusters within $\mathrm{H}$. erato, rather than diverging prior to the $\mathrm{H}$. erato radiation. Red branches indicate rayed lineages. Nodal values are posterior probabilities of clade support, with bold braches indicating a node with >95\% support. Sample names (ID, hybrid zone, race) are indicated at the terminal nodes, color-coded by race

color pattern (Fig. 2). Similar to previous research, there is a strong phylogenetic break across the Andes, with taxa on the eastern slopes of the Andes clustering separately from taxa on the western slopes [32, 47]. All five $H$. himera individuals fall on a well-differentiated lineage that clusters with $H$. erato races from the eastern slopes of the Andes and thus within the $H$. erato radiation (Fig. 2).
Given the long branch length and weaker support values leading to the $H$. himera lineage, we used additional methods to assess the support for H. himera evolving from within the $H$. erato radiation versus predating it. We reran the Bayesian analysis with invariant sites retained, but filtered out sites with too much missing data. The tree topology did not change, nor did the long branch lengths leading to the himera 
lineage (results not shown). The splits tree shows $H$. himera as a strongly monophyletic lineage (i.e. with a long branch length preceding it) and nested within the $H$. erato radiation, with closer affinity for the $H$. erato taxa from the eastern slopes of the Andes than the taxa from the western slopes (Additional file 2: Figure S1). These network plots suggest there may be some phylogenetic conflict arising from allele sharing between $H$. himera and H. e. cyrbia, as evidenced by some of the alternative connections at the base of the topology. However, the combined evidence strongly allies H. e. cyrbia to the western lineage, distinctly separate from $H$. himera, with a low signal of genetic admixture between the two. Collectively, our analyses consistently support $H$. himera as part of the $H$. erato radiation, with a close association to $H$. erato samples from east of the Andes. This placement of $H$. himera was further supported by the phylogenetic reconstructions using a relaxed molecular clock in BEAST. The relaxed molecular clock showed a much better fit to the data than a strict clock (Additional file 3: Figure S2). This suggests that the long branches may be due to a more rapid accumulation of substitutions in the $H$. himera lineage relative to $H$. erato.

A very different phylogenetic history was observed across the genomic region that controls red color pattern variation, reflecting the evolution of the color pattern alleles. This tree strongly clusters taxa by color pattern, showing two reciprocally monophyletic clades sorting perfectly by red phenotype (Fig. 3). The "postman" clade contains all of the $H$. erato postman races, including $H$. erato cyrbia, while the "rayed" clade contains all of the $H$. erato rayed races and $H$. himera. Even though $H$. himera does not have the characteristic rays, it is phenotypically more similar to the rayed phenotypes since they share a number of wing color pattern elements, including the presence of red on the hindwing. This classification of $H$. himera as being most similar to the $H$. erato rayed is supported by our phylogenetic analysis.

While the analyses at loci unlinked to color pattern and across the genomic region containing the functional variants show different patterns of clustering (by geography and phenotype, respectively), both place $H$. himera within the $H$. erato radiation, supporting $H$. himera as an incipient species derived from within $H$. erato. For the incipient species pair, H. himera and H. e. cyrbia, each fall into different monophyletic lineages at both color pattern-linked and unlinked loci. For unlinked loci, H. e. cyrbia is in the western clade and H. himera is in the eastern clade, and for the color locus, H. e. cyrbia falls with the postman races and $H$. himera with the rayed races. From an experimental perspective, the presence of two well-supported monophyletic lineages for each of these analyses suggests that taxa pairs sampled from each of these lineages are expected to have comparable divergence times to the incipient species pair. This enables us to make comparisons between levels of genomic divergence for the incipient species pair and other taxa pairs from the two lineages without having to control for divergence times.

\section{Selection drives divergence at color pattern loci}

Our analysis of relative divergence shows distinct peaks at the functional regions that modulate adaptive differences in wing color patterns between hybridizing $H$. erato races (Fig. 4a). As previously demonstrated, when comparing between parapatric postman and rayed races, there are two distinct elevated peaks of divergence surrounded by steep drop-offs almost all the way to the near zero genomic baseline divergence [31]. These peaks are in sharp contrast to (i) the near zero divergence observed across the red color pattern locus between parapatric races that share similar red color patterns (parapatric postman versus postman comparison, Fig. 4a) and (ii) the broad elevated relative divergence between the incipient species that has no clear peak (parapatric between species comparison, Fig. 4a). We believe that these peaks of divergence are being driven by strong selection on wing color pattern. The similarity of the peaks between the $H$. erato hybrid zones demonstrates the ability of selection to drive similar patterns of divergence among different populations, when the populations are under similar selective pressures.

All of the comparisons between non-hybridizing allopatric races show peaks of relative divergence around the locus modulating red color pattern variation, including comparisons between races that share the same red phenotype (Fig. 4b). The pattern of divergence among allopatric postman versus postman is particularly noteworthy, as it shows strong divergence peaks similar to those observed between hybridizing races with different red color patterns. Similar peaks of relative divergence are also found in the allopatric rayed versus rayed comparisons, albeit the peaks are small. These peaks of relative divergence between similar colored, allopatric races could reflect the history of strong selection at each hybrid zone against the introgression of divergent color patterns. This strong selection on color pattern will also affect allele frequencies at neutral loci that are tightly linked to sites that modulate the color pattern variation. We propose that selection at each hybrid zone may have lead to the fixation of different neutral alleles near the causative sites of the red locus, at each hybrid zone.

Comparisons between patterns of absolute and relative divergence can be used to better understand the role that gene flow and selection play in generating patterns of genomic divergence [48]. Peaks of relative divergence may not always result from divergent selection between hybridizing groups. High relative divergence between 


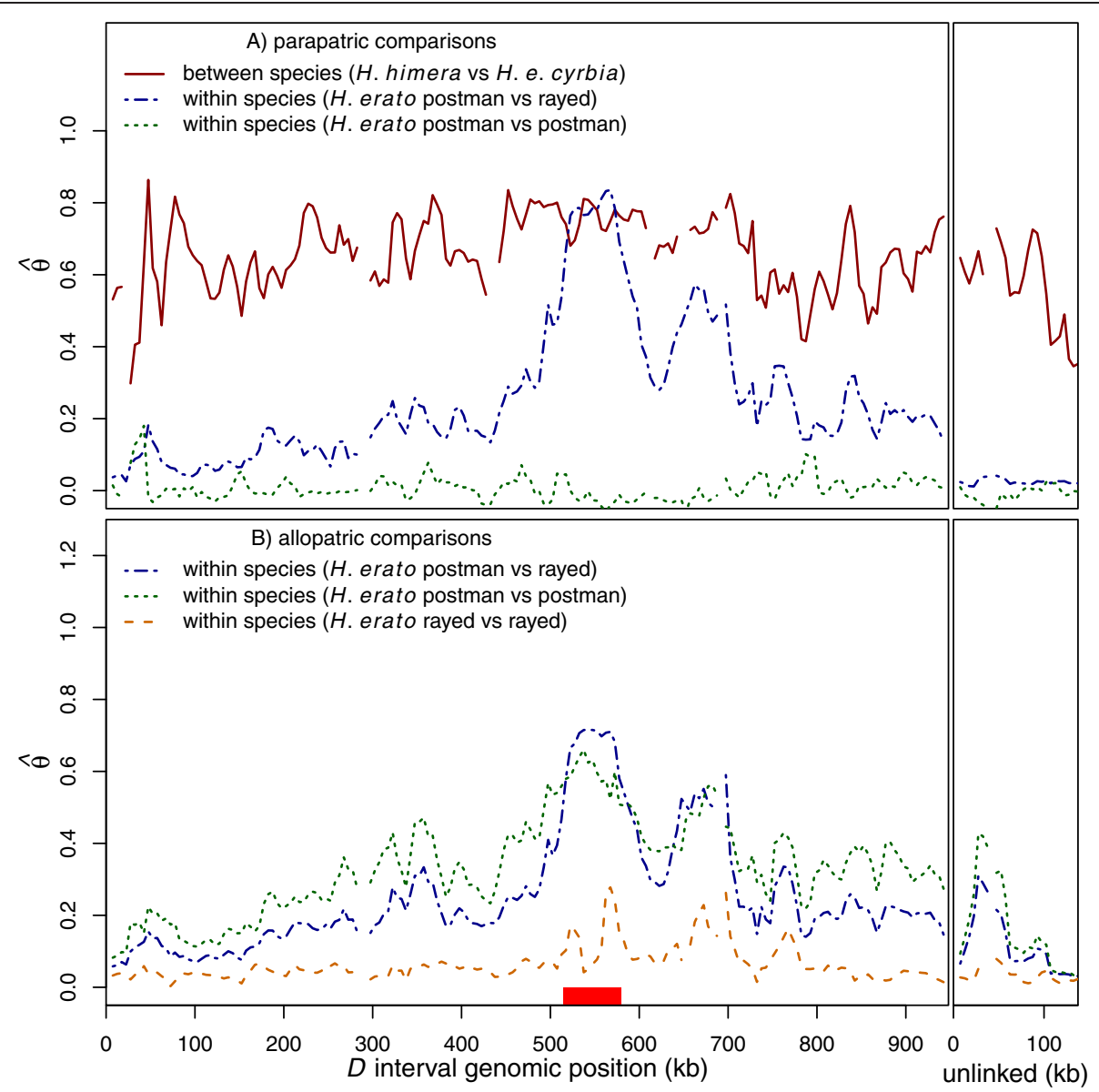

Fig. 4 Relative genomic divergence across the red color pattern $(D)$ interval and unlinked loci. Sliding window (15-kb window size, 5-kb step size) relative genomic divergence across the red $(D)$ interval and genomic regions unlinked to color pattern. The box on the $x$-axis indicates the previously identified 65-kb functional region modulating red color pattern variation [31]. See Table S2 for the taxa pairs included in each comparison, samples sizes, and estimates of baseline divergence from intervals unlinked to color pattern. a Parapatric comparisons show elevated divergence between the incipient species (solid line) at the color pattern locus and loci unlinked to color pattern. The within species comparisons between divergent taxa show a large peak of divergence at the functional region and low baseline divergence (dotted-dashed line). The within species comparisons between taxa with identical red phenotypes show low divergence throughout, with no discernable peaks of divergence (dotted line). b The allopatric within species comparisons show peaks of divergence at the functional region between both divergent taxa and mimetic taxa, with the rayed versus rayed comparison (dashed line) showing much smaller peaks

two groups can also be the result of reduced diversity due to selection within the populations, even in the absence of gene flow $[49,50]$. Overall we found strikingly similar patterns of relative and absolute divergence in each of the hybrid zones. However, absolute divergence was noisier than relative divergence in all comparisons (Additional file 4: Figures S3 and Fig. 4). For all hybridizing taxa that differ in red pattern, there was a clear peak of absolute and relative divergence near the red locus (Additional file 4: Figure S3A). This was expected since we know that all the hybridizing taxa experience ongoing gene flow and strong divergent selection on red wing color patterns. Between the non-hybridizing taxa, the patterns of relative and absolute divergence were not as consistent. Between the allopatric postman races, there were clear peaks of relative and absolute divergence at the color pattern locus (Additional file 4: Figure S3C). These peaks between similarly colored allopatric races likely reflect the history of selection and genetic hitchhiking at each hybrid zone. For example, selection against the introgression of the postman haplotype at the red locus from the postman race into the rayed race in a hybrid zone could result in genetic hitchhiking of neutral alleles at several sites near the red locus. Similarly, selection against the introgression of the postman haplotype at the red locus from the postman race into the rayed race in a different hybrid zone could result in genetic hitchhiking of neutral 
alleles at several sites near the red locus that could be different from the sites that experienced genetic hitchhiking in the first hybrid zone. This could result in a relative excess of allelic differences at several sites near the red locus between the similarly colored races at the two hybrid zones, as we observed in the allopatric postman comparisons. However, this cannot explain the allopatric rayed race comparisons where we found a reduction of absolute divergence near the red locus (Additional file 4: Figure S3D), and only a slight peak of relative divergence (Fig. 4b). These patterns may reflect the relatively recent origin of the rayed phenotype [32] and/or the greater potential for gene flow among the rayed races due to their contiguous distribution across the Amazon basin [51].

Collectively, these results add to a growing body of evidence that demonstrate that selection within populations can leave the same signature of selection as ecological divergence between populations [48, 52, 53]. Given the complex patterns of divergence resulting from the interactions between various evolutionary forces, peaks of divergence should be interpreted with caution, especially when additional supporting data are not available.

\section{Divergence accumulates rapidly during speciation}

The phylogenetic placement of $H$. himera within the broader $H$. erato radiation allows us to compare patterns of genomic divergence at different points along the speciation continuum. Genomic divergence between the incipient species $H$. himera and $H$. e. cyrbia is substantially higher than the divergence seen in all other comparisons. The divergence is much higher across the 1-MB interval, except at the narrow region containing the functional variation where most comparisons show very high divergence (Fig. 4). The unlinked loci also show high divergence between the incipient species (average $=$ $0.519,95 \% \mathrm{CI}=0.507-0.531$ ), significantly higher than any of the within species comparisons (maximum average $=0.191,95 \% \mathrm{CI}=0.182-0.200$ ) (Additional file 1: Table S2). This is a relatively high level of divergence given that $H$. himera is a recent incipient species and is known to hybridize frequently with $H$. erato in nature (Figs. 2 and 3, [16-18]). These patterns suggest that divergence may accumulate rapidly in the early stages of speciation, even if reproductive isolation is incomplete and gene flow is still occurring. Some previous work in other Heliconius clades has also shown that genomic divergence can occur rapidly as speciation progresses [54, 55]; however, one study of other hybridizing Heliconius species suggested divergence may build gradually over long periods of continuous gene flow [56]. Our results are more consistent with the former Heliconius studies, as well as empirical studies in other systems that report high genome wide divergence early in speciation, even in the face of ongoing gene flow $[57,58]$.
What drives high divergence between incipient species? Our experimental design allows us to begin to disentangle the effects of selection and gene flow on patterns of genomic divergence during speciation. By comparing levels of divergence between hybridizing and non-hybridizing races, we can empirically see the effects of differing levels of gene flow. As expected, non-hybridizing races (allopatric) show higher background levels of genomic divergence than hybridizing races (parapatric) (Additional file 5: Figure S4, blue shaded area [0.042-0.173] versus green shaded area [0.020-0.031]). Comparisons of non-hybridizing races provide an estimate of the expected level of divergence between divergently colored taxa with limited gene flow. Since, the phylogenetic reconstructions showed that $H$. himera and $H$. cyrbia belong to different clades at the color locus and color unlinked loci, we may expect similar levels of genomic divergence to have accumulated between the incipient species, as have accumulated between nonhybridizing races from the two different clades.

In contrast to this expectation, genomic divergence between the incipient species is much higher than between the non-hybridizing races (Fig. 4 and Additional file 5: Figure S4). Divergence between the incipient species was not only higher across the color pattern locus, but also across the genomic regions unlinked to color pattern variation. For instance, the background divergence between non-hybridizing races with different color patterns from Peru and French Guiana (0.191; 95 \% CI: 0.1820.200 ) is significantly lower than the divergence between the incipient species (0.519; $95 \%$ CI: 0.507-0.531), despite higher realized rates of gene flow between the incipient species than the non-hybridizing races. $H$. himera and $H$. e. cyrbia hybridize continuously and multi-generational hybrids (based on color pattern genotypes) can readily be found where they overlap geographically. This suggests that there is likely genomic admixture occurring every generation between the incipient species. In contrast, the non-hybridizing races sampled are often thousands of kilometers apart (e.g. Peru and French Guiana are located on opposite sides of South America) and never come into direct contact. Even though $H$. erato does have a wide, continuous distribution across South America, the lifetime dispersal distance for $H$. erato is estimated to be only $\sim 5 \mathrm{~km}$ per generation [59], which suggests admixture between races thousands of kilometers apart would take hundreds of generations to occur. Therefore, despite the expectation that higher gene flow should homogenize genetic variation and decrease divergence across the genome, we find that divergence is higher between the taxa that also have greater potential for gene flow.

The higher levels of divergence between $H$. himera and H. e. cyrbia likely reflect unique selective pressures and/or population histories. These differences could simply be 
the result of population bottlenecks and/or periods of allopatry that allowed divergence to rapidly accumulate between the populations, and the hybridization is due to recent secondary contact. Alternatively, these patterns could be the result of natural selection rapidly driving genomic divergence, even with continuous gene flow. Selection has been shown to play a primary role in speciation in both theoretical models [60] and empirical studies [see references in 1]. In particular, selection acting on many loci across the genome is suggested to result in an increase in genome wide divergence [61,62]. Unlike geographic races of $H$. erato, $H$. himera and $H$. e. cyrbia show strong divergence beyond just color pattern, including differences in mating preference, larval developmental time, adult physiology, and habitat preferences [14-18, 63, 64]. Our results support the hypothesis that divergent selection on multiple traits and loci may drive the high divergence found between the incipient species $H$. himera and $H$. $e$. cyrbia. Our within species comparisons show that the effect of selection on color pattern loci is restricted to a small region of the genome, with the decay of relative divergence occurring between 1-2 $\mathrm{cM}$ from the causative locus (Additional file 5: Figure S4). This rapid rate of decay is what is predicted from models of divergence hitchhiking around a gene under strong selection [4]. Our results are consistent with several theoretical and empirical studies that report high genome wide divergence and a limited role for divergence hitchhiking in promoting speciation $[54,57,58,61,62,65,66]$. These results contrast with a study in the Heliconius melpomene clade, that suggested that divergence hitchhiking may play a prominent role during the early stages of speciation [56]. However, $H$. erato and $H$. himera show stronger evidence of habitat based ecological divergence than the incipient species studied in the H. melpomene clade [33]. Our results highlight a limited role of divergence hitchhiking and suggest that the increase in divergence seen between the incipient species likely requires genomic hitchhiking through selection at multiple loci.

\section{Conclusions}

Heliconius butterflies provide a full continuum of taxa pairs at varying stages of reproductive isolation-from freely hybridizing color pattern races to completely reproductively isolated species. By sampling across this continuum, we can observe how genomes diverge through the speciation process and begin to disentangle the evolutionary forces that drive species divergence. Here, we resolve the phylogenetic placement of $H$. himera within the $H$. erato clade and demonstrate that $H$. himera shares a similar haplotype at the red color pattern locus to rayed $H$. erato races. This phylogenetic reconstruction demonstrates that the hybridizing color pattern races and incipient species sampled in this study reflect distinct points of divergence along the speciation continuum. Comparisons of population divergence at these different points in the continuum show that as reproductive isolation increases, genomic divergence increases, not only near the regions responsible for adaptive divergence and reproductive isolation, but also at regions unlinked to known adaptive loci. This occurs much earlier in the speciation process than previously envisioned. By examining different regions of the genome, we were able to provide a series of snapshots of divergence through the speciation process that suggest complex population histories and selective pressures likely drive rapid divergence early in the speciation process. The results also suggest that reference-genome-enabled, genome-wide population demographic analyses across the $H$. erato speciation continuum may provide valuable insights into the effects of selection and gene flow on the origin of species.

\section{Availability of supporting data}

The data supporting the results of this article, including SNP calls and phylogenetic trees, are available in the Dryad repository [doi:10.5061/dryad.5n10d, http://datadryad.com]. Aligned sequencing reads are available at the NCBI Short Read Archive [GenBank:SRA059521 (SRX1176360, SRX1176362-3, SRX1176365-74), http:// www.ncbi.nlm.nih.gov/sra]. Custom analysis scripts are available on GitHub [https://github.com/LaMariposa].

\section{Additional files}

Additional file 1: Table S1. Samples and sequencing data. Table S2. Taxa pairs for divergence analysis. (PDF $140 \mathrm{~kb}$ )

Additional file 2: Figure S1. Phylogenetic network at loci unlinked to color pattern. Neighbor-net splits tree network at loci unlinked to color pattern. Terminal nodes indicate voucher number, hybrid zone, and race of each sample. Heliconius himera shows a stronger affinity to taxa east of the Andes than the taxa from west of the Andes, which cluster separately. (PDF $62 \mathrm{~kb}$ )

Additional file 3: Figure S2. Phylogenetic relationships under a relaxed clock. The phylogenetic tree generated when substitution rates among lineages are allowed to vary. The tree shows $\mathrm{H}$. himera nested within the H. erato radiation. Results for Bayesfactor comparison of strict and relaxed molecular clock topologies are shown in upper left corner. (PNG 109 kb)

Additional file 4: Figure $\mathbf{S 3}$. Absolute genomic divergence across the red color pattern $(D)$ interval and unlinked loci. Sliding window (15-kb window size, 5-kb step size) absolute genomic divergence $\left(d_{x y}\right)$ across the red $(D)$ interval and genomic regions unlinked to color pattern. See Additional file 1: Table S2 for the taxa pairs included in each comparison and samples sizes. (A) Parapatric hybrid zones. (B) Allopatric postman versus rayed comparisons. (C) Allopatric postman versus postman comparisons. (D) Allopatric rayed versus rayed comparisons. (PDF $29 \mathrm{~kb}$ )

Additional file 5: Figure S4. Decay of divergence with recombination distance. The curves represent the decay of genomic divergence with distance from the causative color pattern locus between taxa pairs with divergent phenotypes. We converted genomic distance (bp) to recombination distance $(\mathrm{CM})$ assuming a constant recombination rate based the $H$. erato linkage map size (1430 cM) [45] and the estimated 
size of the $\mathrm{H}$. erato genome (400 Mb) [46], thus assuming a constant recombination rate across the genomic regions and across taxa. The extent of variation in recombination rate in Heliconius is unknown, as such this estimate merely provides a rough estimate of recombination distance from the causative locus. See Additional file 1: Table S2 for the taxa pairs included in each comparison, samples sizes, and estimates of baseline divergence from intervals unlinked to color pattern. The results were loess smoothed for presentation, which potentially introduces additional biases in the estimates of rate of decay. The comparisons are between incipient species (H. himera versus H. e. cyrbia; red dashed), average of within species parapatric postman versus rayed pairs (green dotted-dashed), and average of within species allopatric postman versus rayed pairs (blue dotted). The horizontal red dashed line represents the background genomic divergence between the incipient species at loci unlinked to color pattern. The blue and green shaded boxes are the range of baseline divergences at unlinked loci for within species allopatric and parapatric comparisons, respectively. The divergence at both the color pattern locus and unlinked genomic regions is substantially higher between species than it is within species. (PDF $5 \mathrm{~kb}$ )

\section{Competing interests}

The authors declare that they have no competing interests.

\section{Authors' contributions}

Experimental design is credited to BAC, RP, MAS, WOM, HMH; data collection was carried out by MAS, BAC, WOM; data analysis was done by MAS, BAC, $\mathrm{HMH}$; the manuscript was prepared by MAS, BAC, with input from all authors. All authors have read and approved the final manuscript.

\section{Authors' information}

Not applicable.

\section{Acknowledgements}

We would like to thank Diana Outlaw for assistance with phylogenetic analyses (BEAST) and Jenny Barna and Chris Smith for computational support. We would also like to thank Jeff Feder and two anonymous reviewers for comments that greatly improved the manuscript. Samples were collected with permission from Ecuador's Ministerio del Ambiente (006-2012-IC-FAU-DPL-MA, 005-13 IC-FAU-DNB/MA, 013-09 IC-FAU-DNB/ MA); Peru's Ministerio de Agricultura and Instituto Nacional de Recursos Naturales (004-2008-INRENAIFFS-DCB, 011756-AG-INRENA); French Guiana's Ministere de L'Ecologie, de L'Energie, du Developpemet Durable et de laMar (BIODAD-2010-0433); Panama's Autoridad Nacional del Ambiente (SC/A-7-11).

\section{Funding}

This work was funded by the following awards: Hanne and Torkel Weis-Fogh Fund (sample collection, awarded to Nicola Nadeau and Richard Merrill); CNRS Nouraugues (BAC); NSF DEB-1257839 (BAC), DEB-1257689 (WOM), DEB1027019 (WOM); and the Smithsonian Institution.

\section{Author details}

${ }^{1}$ Smithsonian Tropical Research Institute, Panamá City, Panamá.

${ }^{2}$ Biomathematics Program, North Carolina State University, Raleigh, NC 27695, USA. ${ }^{3}$ Research School of Biology, The Australian National University, 134 Linnaeus Way, Canberra, ACT 2601, Australia. ${ }^{4}$ Department of Biology and Center for Applied Tropical Ecology and Conservation, University of Puerto Rico-Rio Piedras, 00931 San Juan, Puerto Rico. ${ }^{5}$ Department of Biology, Pennsylvania State University, 208 Mueller Laboratory, University Park, PA 16802, USA. ${ }^{6}$ Department of Biological Sciences, Mississippi State University, 295 Lee Boulevard, Mississippi State, MS 39762, USA.

\section{Received: 20 March 2015 Accepted: 14 September 2015}

\section{Published online: 24 September 2015}

\section{References}

1. Nosil P. Ecological Speciation. Oxford University Press; 2012.

2. Harrison RG. The language of speciation. Evolution. 2012;66:3643-57.

3. Nosil P, Funk DJ, Ortiz-Barrientos D. Divergent selection and heterogeneous genomic divergence. Mol Ecol. 2009;18:375-402.
4. Feder JL, Egan SP, Nosil P. The genomics of speciation-with-gene-flow. Trends Genet. 2012;28:342-50.

5. Mallet J. Speciation, raciation, and color pattern evolution in Heliconius butterflies: evidence from hybrid zones. In: Harrison RG, editor. Hybrid zones and the evolutionary process. New York, NY: Oxford University Press; 1993. p. 226-60

6. Jiggins CD, Estrada C, Rodrigues A. Mimicry and the evolution of premating isolation in Heliconlus melpomene Linnaeus. J Evol Biol. 2004;17:680-91.

7. Gilbert LE. Biodiversity of a Central American Heliconius community: pattern, process, and problems. In: Price P, Lewinsohn T, Fernandes T, Benson W, editors. Plant-animal interactions: evolutionary ecology in tropical and temperate regions. New York, NY: John Wiley and Sons; 1991. p. 403-27.

8. Mallet J. The genetics of warning colour in Peruvian hybrid zones of Heliconius erato and $\mathrm{H}$. melpomene. Proc R Soc London Ser B Biol Sci. 1989;236:163-85

9. Mallet J, Barton N, Lamas G, Santisteban J, Muedas M, Eeley H. Estimates of selection and gene flow from measures of cline width and linkage disequilibrium in Heliconius hybrid zones. Genetics. 1990;124:921-36.

10. Mallet J. Hybrid zones of Heliconius butterflies in Panama and the stability and movement of warning colour clines. Heredity. 1986;56:191-202.

11. Blum MJ. Ecological and genetic associations across a Heliconius hybrid zone. J Evol Biol. 2008;21:330-41.

12. Blum MJ. Rapid movement of a Heliconius hybrid zone: evidence for phase III of Wright's shifting balance theory? Evolution. 2002;56:1992-8.

13. Mallet J, Barton N. Strong natural selection in a warning-color hybrid zone. Evolution. 1989;43:421-31.

14. McMillan WO, Jiggins CD, Mallet J. What initiates speciation in passion-vine butterflies? Proc Natl Acad Sci U S A. 1997;94:8628-33.

15. Merrill RM, Chia A, Nadeau NJ. Divergent warning patterns contribute to assortative mating between incipient Heliconius species. Ecol Evol. 2014:4:911-7.

16. Mallet J, McMillan W, Jiggins C. Estimating the mating behavior of a pair of hybridizing Heliconius species in the wild. Evolution. 1998;52:503-10.

17. Descimon H, Mast de Maeght J. Semispecies relationships between Heliconius erato cyrbia Godt. and H. himera Hew. in Southwestern Ecuador. J Res Lepid. 1984;22:229-37.

18. Jiggins CD, McMillan WO, Neukirchen W, Mallet J. What can hybrid zones tell us about speciation? The case of Heliconius erato and $\mathrm{H}$. himera (Lepidoptera: Nymphalidae). Biol J Linn Soc. 1996;59:221-42.

19. Brower AVZ. Rapid morphological radiation and convergence among races of the butterfly Heliconius erato inferred from patterns of mitochondrial DNA evolution. Proc Natl Acad Sci U S A. 1994;91:6491-5.

20. Jiggins C, McMillan W, King P, Mallet J. The maintenance of species differences across a Heliconius hybrid zone. Heredity. 1997;79:495-505.

21. Flanagan NS, Tobler A, Davison A, Pybus OG, Kapan DD, Planas S, et al. Historical demography of Müllerian mimicry in the neotropical Heliconius butterflies. Proc Natl Acad Sci U S A. 2004;101:9704-9.

22. Joron M, Frezal L, Jones RT, Chamberlain NL, Lee SF, Haag CR, et al. Chromosomal rearrangements maintain a polymorphic supergene controlling butterfly mimicry. Nature. 2011;477:203-6.

23. Reed RD, Papa R, Martin A, Hines HM, Counterman BA, Pardo-Diaz C, et al. optix drives the repeated convergent evolution of butterfly wing pattern mimicry. Science. 2011;333:1137-41.

24. Martin A, Papa R, Nadeau NJ, Hill Rl, Counterman BA, Halder G, et al. Diversification of complex butterfly wing patterns by repeated regulatory evolution of a Wnt ligand. Proc Natl Acad Sci U S A. 2012;109:12632-7.

25. Papa R, Kapan DD, Counterman BA, Maldonado K, Lindstrom DP, Reed RD, et al. Multi-allelic major effect genes interact with minor effect QTLs to control adaptive color pattern variation in Heliconius erato. PLoS One. 2013;8, e57033.

26. Ferguson L, Lee SF, Chamberlain N, Nadeau N, Joron M, Baxter S, et al. Characterization of a hotspot for mimicry: assembly of a butterfly wing transcriptome to genomic sequence at the HmYb/Sb locus. Mol Ecol. 2010;19:240-54.

27. Counterman BA, Araujo-Perez F, Hines HM, Baxter SW, Morrison CM, Lindstrom DP, et al. Genomic hotspots for adaptation: the population genetics of Müllerian mimicry in Heliconius erato. PLoS Genet. 2010;6, e1000796.

28. Baxter SW, Nadeau NJ, Maroja LS, Wilkinson P, Counterman BA, Dawson A, et al. Genomic hotspots for adaptation: the population genetics of Müllerian mimicry in the Heliconius melpomene clade. PLoS Genet. 2010;6, e1000794. 
29. Sheppard PM, Turner JRG, Brown KS, Benson WW, Singer MC. Genetics and the evolution of Mullerian mimicry in Heliconius butterflies. Philos Trans R Soc London Ser B, Biol. 1985;308:433-610.

30. Papa R, Martin A, Reed RD. Genomic hotspots of adaptation in butterfly wing pattern evolution. Curr Opin Genet Dev. 2008;18:559-64.

31. Supple MA, Hines HM, Dasmahapatra KK, Lewis JJ, Nielsen DM, Lavoie C, et al. Genomic architecture of adaptive color pattern divergence and convergence in Heliconius butterflies. Genome Res. 2013;23:1248-57.

32. Hines HM, Counterman BA, Papa R, Albuquerque de Moura P, Cardoso MZ, Linares $M$, et al. Wing patterning gene redefines the mimetic history of Heliconius butterflies. Proc Natl Acad Sci U S A. 2011;108:19666-71.

33. Mallet J, McMillan W, Jiggins C. Mimicry and warning color at the boundary between races and species. In: Howard DJ, Berlocher SH editors. Endless forms: species and speciation. Oxford: University Press; 1998. p. 390-403.

34. Li H, Durbin R. Fast and accurate short read alignment with BurrowsWheeler transform. Bioinformatics. 2009;25:1754-60.

35. McKenna A, Hanna M, Banks E, Sivachenko A, Cibulskis K, Kernytsky A, et al The genome analysis toolkit: a MapReduce framework for analyzing nextgeneration DNA sequencing data. Genome Res. 2010;20:1297-303.

36. DePristo MA, Banks E, Poplin R, Garimella KV, Maguire JR, Hartl C, et al. A framework for variation discovery and genotyping using next-generation DNA sequencing data. Nat Genet. 2011:43:491-8.

37. Swofford D. PAUP*. Phylogenetic Analysis Using Parsimony (*and Other Methods). Version 4. Sunderland, MA: Sinauer Associates; 2002.

38. Nylander JAA. MrModeltest v2. 2004.

39. Ronquist F, Teslenko M, van der Mark P, Ayres DL, Darling A, Höhna S, et al. MrBayes 3.2: efficient Bayesian phylogenetic inference and model choice across a large model space. Syst Biol. 2012;61:539-42.

40. Rambaut A, Suchard M, Drummond A. Tracer v1.6. 2013.

41. Huson DH, Bryant D. Application of phylogenetic networks in evolutionary studies. Mol Biol Evol. 2006:23:254-67.

42. Drummond AJ, Suchard MA, Xie D, Rambaut A. Bayesian phylogenetics with BEAUti and the BEAST 1.7. Mol Biol Evol. 2012;29:1969-73.

43. Weir BS. Genetic Data Analysis II. Sunderland, MA: Sinauer Associates; 1996.

44. Nei M. Molecular Evolutionary Genetics. Columbia University Press; 1987.

45. Kapan DD, Flanagan NS, Tobler A, Papa R, Reed RD, Gonzalez JA, et al. Localization of Müllerian mimicry genes on a dense linkage map of Heliconius erato. Genetics. 2006;173:735-57.

46. Tobler A, Kapan D, Flanagan NS, Gonzalez C, Peterson E, Jiggins CD, et al. First-generation linkage map of the warningly colored butterfly Heliconius erato. Heredity. 2005;94:408-17.

47. Quek SP, Counterman BA, Albuquerque de Moura P, Cardoso MZ, Marshall CR, McMillan WO, et al. Dissecting comimetic radiations in Heliconius reveals divergent histories of convergent butterflies. Proc Natl Acad Sci U S A. 2010;107:7365-70

48. Cruickshank TE, Hahn MW. Reanalysis suggests that genomic islands of speciation are due to reduced diversity, not reduced gene flow. Mol Ecol 2014:23:3133-57.

49. Charlesworth B. Measures of divergence between populations and the effect of forces that reduce variability. Mol Biol Evol. 1998;15:538-43.

50. Charlesworth B, Nordborg M, Charlesworth D. The effects of local selection, balanced polymorphism and background selection on equilibrium patterns of genetic diversity in subdivided populations. Genet Res. 1997;70:155-74.

51. Rosser N, Phillimore A, Huertas B, Willmott KR, Mallet J. Testing historical explanations for gradients in species richness in heliconiine butterflies of tropical America. Biol J Linn Soc. 2012;105:479-97.

52. Noor MAF, Bennett SM. Islands of speciation or mirages in the desert? Examining the role of restricted recombination in maintaining species. Heredity. 2009;103:439-44.

53. Nachman MW, Payseur BA. Recombination rate variation and speciation: theoretical predictions and empirical results from rabbits and mice. Philos Trans R Soc B Biol Sci. 2012;367:409-21.

54. Kronforst MR, Hansen MEB, Crawford NG, Gallant JR, Zhang W, Kulathinal RJ, et al. Hybridization reveals the evolving genomic architecture of speciation. Cell Rep. 2013:5:666-77.

55. Martin SH, Dasmahapatra KK, Nadeau NJ, Salazar C, Walters JR, Simpson F, et al. Genome-wide evidence for speciation with gene flow in Heliconius butterflies. Genome Res. 2013;23:1817-28.

56. Nadeau NJ, Whibley A, Jones RT, Davey JW, Dasmahapatra KK, Baxter SW, et al. Genomic islands of divergence in hybridizing Heliconius butterflies identified by large-scale targeted sequencing. Philos Trans R Soc Lond B Biol Sci. 2012;367:343-53.

57. Michel A, Sim S, Powell THQ, Taylor MS, Nosil P, Feder JL. Widespread genomic divergence during sympatric speciation. Proc Natl Acad Sci U S A. 2010;107:9724-9.

58. Lawniczak MKN, Emrich SJ, Holloway AK, Regier AP, Olson M, White B, et al. Widespread divergence between incipient Anopheles gambiae species revealed by whole genome sequences. Science. 2010;330:512-4

59. Mallet J. Dispersal and gene flow in a butterfly with home range behavior: Heliconius erato (Lepidoptera, Nymphalidae). Oecologia. 1986;68:210-7.

60. Gavrilets S. Waiting time to parapatric speciation. Proc Biol Sci. 2000;267:2483-92.

61. Feder JL, Gejji R, Yeaman S, Nosil P. Establishment of new mutations under divergence and genome hitchhiking. Philos Trans R Soc Lond B Biol Sci. 2012;367:461-74

62. Flaxman SM, Feder JL, Nosil P. Genetic hitchhiking and the dynamic buildup of genomic divergence during speciation with gene flow. Evolution. 2013;67:2577-91

63. Davison A, McMillan WO, Griffin AS, Jiggins CD, Mallet JLB. Behavioral and physiological differences between two parapatric Heliconius species. Biotropica. 1999;31:661-8.

64. Pardo-Diaz C, Salazar C, Baxter SW, Merot C, Figueiredo-Ready W, Joron M, et al. Adaptive introgression across species boundaries in Heliconius butterflies. PLoS Genet. 2012;8, e1002752.

65. Feder $\mathrm{L}$, Nosil P. The efficacy of divergence hitchhiking in generating genomic islands during ecological speciation. Evolution. 2010;64:1729-47.

66. Andrew RL, Rieseberg LH. Divergence is focused on few genomic regions early in speciation: incipient speciation of sunflower ecotypes. Evolution. 2013;67:2468-82

\section{Submit your next manuscript to BioMed Central and take full advantage of:}

- Convenient online submission

- Thorough peer review

- No space constraints or color figure charges

- Immediate publication on acceptance

- Inclusion in PubMed, CAS, Scopus and Google Scholar

- Research which is freely available for redistribution 